Research Article

\title{
Inverse Analysis of the Partitioning Deformation Modulus of High-Arch Dams Based on Quantum Genetic Algorithm
}

\author{
Yantao Zhu $\mathbb{D}^{1,2,3}$ Xinqiang Niu, ${ }^{4,5}$ Jimin Wang, ${ }^{6}$ Chongshi Gu $\mathbb{D}^{1,2,3}$ Erfeng Zhao, ${ }^{1,2,3}$ \\ and Lixian Huang ${ }^{7}$ \\ ${ }^{1}$ State Key Laboratory of Hydrology-Water Resources and Hydraulic Engineering, Hohai University, Nanjing, China \\ ${ }^{2}$ National Engineering Research Center of Water Resources Efficient Utilization and Engineering Safety, Hohai University, \\ Nanjing, China \\ ${ }^{3}$ College of Water Conservancy and Hydropower Engineering, Hohai University, Nanjing 210098, China \\ ${ }^{4}$ Changjiang Institute of Survey, Planning, Design and Research, Wuhan, Hubei 430010, China \\ ${ }^{5}$ National Dam Safety Research Center, Wuhan 430010, China \\ ${ }^{6}$ Yalong River Hydropower Development Company, LTD., Chengdu 610051, China \\ ${ }^{7}$ Materials Science and Engineering Department, University of California Los Angeles, Los Angeles, CA 90095, USA
}

Correspondence should be addressed to Yantao Zhu; zyt50@163.com and Chongshi Gu; csgu@hhu.edu.cn

Received 30 December 2019; Revised 15 March 2020; Accepted 2 September 2020; Published 29 September 2020

Academic Editor: Claudio Mazzotti

Copyright (c) 2020 Yantao Zhu et al. This is an open access article distributed under the Creative Commons Attribution License, which permits unrestricted use, distribution, and reproduction in any medium, provided the original work is properly cited.

The physical and mechanical parameters of hydraulic structures in complicated operating conditions often change over time. Updating these parameters in a timely manner is important to comprehend the operating behaviors and monitor the safety of hydraulic structures. Conventional inverse analysis methods can only generate inversions on the comprehensive deformation modulus of concrete dam structures, which contradict practical conditions. Based on the researches on conventional reversion methods of the deformation modulus of the dam body, foundation, and reservoir basin, the objective fitness function is established in this paper according to engineering-measured data and finite element simulation results. The quantum genetic algorithm has high global search efficiency and population diversity. A mechanical parameter inversion of high-arch dams is built from the intelligent optimization of an established algorithm by applying the quantum genetic algorithm. The proposed algorithm is tested to be feasible and valid for practical engineering projects and therefore shows scientific and practical application values.

\section{Introduction}

Hydraulic structural designs and the stress-strain and crack formation mechanisms of engineering structures are largely determined by the physical and mechanical parameters of the geotechnical engineering medium. Parameter selection can reflect and test the design and construction of hydraulic structures. The physical and mechanical parameters of higharch dams often change with time. This variation is attributed to complicated and diversified service conditions. Hence, updating these parameters in a timely manner is highly important for the security monitoring of concrete gravity dams. In addition, the designed physical and mechanical parameters differ from the actual situations to some extent because of uncertain design parameters, calculation models, and boundary conditions. The difference can also be attributed to the calculation loads and working conditions, construction quality, and geological conditions. To comprehend the actual deformation behaviors of high-arch dams, the deformation of the physical and mechanical parameters under the actual state should be determined through parameter inversion based on measured data [1-3].

Based on the results of a positive analysis, some in situ monitoring information is used to trace back the physical and mechanical parameters of the dam. This process is called the inversion analysis of physical and mechanical parameters of the dam. Chen [4] used the monitoring data of seepage pressure gauges to obtain the diffusion coefficient of dam 
concrete. $\mathrm{Wu}$ and Ruan [5] used deformation monitoring data to invert the comprehensive elastic modulus of dam body, the linear expansion coefficient, and the comprehensive deformation modulus of dam foundation, and the proposed method has been widely used in practical projects. $\mathrm{Gu}$ and $\mathrm{Wu}$ [6] proposed an inverse analysis model for the viscous coefficient of dam body and foundation by using viscoelastic finite elements and combining with the in situ monitoring data of the deformation. They also derived the inversion formula of the variable tolerance method for the viscous coefficient. Su and Peng [7] after fully considering the influence of uncertainty on the inversion results of physical and mechanical parameters introduced the interval analysis method to process in situ monitoring data and finite element calculation results and discussed the inversion model of the comprehensive elastic modulus of the concrete dam body and the comprehensive deformation modulus of the dam foundation rock body.

The inverse analysis is typically a complicated nonlinear function optimization problem. The use of a global optimization algorithm with rich theoretical experience is the approach to ideal intelligent optimization. Friswell et al. [8] determined structural damages using the genetic algorithm. Li et al. [9] realized the intelligent optimization of Earth's physical and mechanical parameters using the genetic algorithm. Li et al. [10] proposed the inversion algorithm of the initial stress field of rock based on the genetic algorithm. Their group then implemented inversion analysis on the parameters of the corresponding rock and concrete material. Guo et al. [11] studied the multisectional analysis algorithm of the compensation monitoring information on the basis of the monitoring data. They also established the genetic optimization model for parameter inversion. According to the direct random optimization characteristics of the genetic algorithm and the three-dimensional finite element algorithm, Xiang et al. $[12,13]$ proposed the inversion method of the deformation modulus of the dam body and foundation. This approach is employed by combining the security monitoring data of the dam structure (e.g., horizontal displacement). Considering the construction process, the compaction mechanism, and the lay characteristics of a roller-compacted concrete dam, Niu and Shi [14] viewed the identification and inversion problem of mechanical parameters as the optimization process. Then, the author applied inversion to the transverse isotropic deformation and gradient parameters of a roller-compacted concrete dam using the chaos genetic algorithm.

As an emerging computing model, quantum computing is a combination of quantum theory, information theory, and computer science. Based on the quantum state characteristic, quantum computing uses the qubit probability coding to represent the chromosomes. Quantum computing can achieve the objective of the objective function for optimization through the role of the quantum revolving door of constant update to update and optimize the population, so it has high efficiency in global searching and good species diversity. Genetic algorithm is a heuristic search algorithm that simulates the evolution mechanism of species in nature. However, since the traditional genetic algorithm involves too much computation to solve some problems, it is difficult to find their optimal solutions correspondingly [15-19]. This prompted researchers to combine quantum theory and genetic algorithm to build a more efficient and fast genetic algorithm.

In this paper, the intelligent optimization of the ideal objective function is realized using the quantum genetic algorithm (QGA), and the portioning QGA inversion model based on the finite element simulation results is constructed using the high-efficiency core solver, Abaqus. The fitness function is established based on the finite element simulation and measured results. The mechanical parameters of the high-arch dam are inverted using MATLAB programming. The proposed algorithm is verified feasible and valid through the inversion of the comprehensive concrete deformation modulus at different positions on the high-arch dam. The research results have certain scientific and practical application values.

\section{Traditional Inversion Analysis Algorithms of the Deformation Modulus of High-Arch Dams}

The material parameters of a high-arch dam for the inversion analysis include the deformation modulus of the dam body $\left(E_{c}\right)$, deformation modulus of the rock dam foundation $\left(E_{r}\right)$, and the deformation modulus of the rock reservoir basin $\left(E_{b}\right)$. In the inversion analysis based on the finite element simulation model, these parameters are all assumed as the initial value. Given the fixed external loads (the hydraulic pressure $H$ is generally used as the external load), the hydraulic pressure component $f_{1}(H, x, y, z)$ obtained by forward modeling must be corrected to achieve displacement under actual hydraulic loads $f_{1}^{\prime}(H, x, y, z)$. In other words,

$$
f_{1}^{\prime}(H, x, y, z)=X f_{1}(H, x, y, z)
$$

$f_{1}^{\prime}(H, x, y, z)$ is the combined displacement of the dam body, the dam foundation, and the reservoir basin. Therefore, when correcting the simulated $f_{1}^{\prime}(H, x, y, z)$, it is obtained simply by adjusting one parameter; that is, $X f_{1}^{\prime}(H, x, y, z)$ can only be gained by adjusting the three regions mentioned above. On this basis, real $E_{c}, E_{r}$, and $E_{b}$ could be deduced.

In the linear deformation range, the overall stiffness matrix in the equilibrium equation of the dam and rock foundation is 


$$
[K]=\sum_{e_{j} \in \Omega_{1}}[C]_{e_{j}}^{\mathrm{T}}[K]_{e_{j}}[C]_{e_{j}}+\sum_{e_{j} \in \Omega_{2}}[C]_{e_{j}}^{\mathrm{T}}[K]_{e_{j}}[C]_{e_{j}}+\sum_{e_{j} \in \Omega_{3}}[C]_{e_{j}}^{\mathrm{T}}[K]_{e_{j}}[C]_{e_{j}} \text {, }
$$

where $[K]_{e_{j}}=\iiint_{\Omega_{j}}[B]^{\mathrm{T}}[D][B] \mathrm{d} \Omega=E \iiint_{\Omega_{j}}[B]^{\mathrm{T}} f(\mu)[B]$
$\mathrm{d} \Omega=E[\bar{K}]_{e_{j}}$ $\mathrm{d} \Omega=E[\bar{K}]_{e_{j}}$.

If $\Omega_{1}, \Omega_{2}$, and $\Omega_{3}$ represent the regions where the dam body, dam foundation, and reservoir basin lay, then

$$
E= \begin{cases}E_{c} & e_{j} \in \Omega_{1}, \\ E_{r} & e_{j} \in \Omega_{2}, \\ E_{b} & e_{j} \in \Omega_{3} .\end{cases}
$$

If $R_{1}=\left(E_{r} / E_{c}\right)$ and $R_{2}=\left(E_{b} / E_{c}\right)$, then

$$
\begin{aligned}
{[K] } & =E_{c} \sum_{e_{j} \in \Omega_{1}}[C]_{e_{j}}^{\mathrm{T}}[K]_{e_{j}}[C]_{e_{j}}+R_{1} \sum_{e_{j} \in \Omega_{2}}[C]_{e_{j}}^{\mathrm{T}}[K]_{e_{j}}[C]_{e_{j}}+R_{2} \sum_{e_{j} \in \Omega_{3}}[C]_{e_{j}}^{\mathrm{T}}[K]_{e_{j}}[C]_{e_{j}} \\
& =F\left(\frac{E_{c}, E_{r}}{\left(E_{c}, E_{b} / E_{c}\right)}\right), f\left(\mu_{c}\right), f\left(\mu_{r}\right), f\left(\mu_{b}\right), L, S,
\end{aligned}
$$

where $E_{c}, E_{r}$, and $E_{b}$ are the deformation moduli of the dam body, dam foundation, and reservoir basin, respectively; $\mu_{c}$, $\mu_{r}$, and $\mu_{b}$ are Poisson's ratios of the dam body, dam foundation, and reservoir basin, respectively; $f\left(\mu_{c}\right), f\left(\mu_{r}\right)$, and $f\left(\mu_{b}\right)$ are the variables related to Poisson's ratios of the dam body, dam foundation, and reservoir basin, respectively; and $L$ and $S$ reflect the unit size and influences of the constructs in the calculation region.

Equation (4) shows that the stiffness matrix $[K]$ is influenced by $E_{c},\left(E_{r} / E_{c}\right),\left(E_{b} / E_{c}\right), f\left(\mu_{c}\right), f\left(\mu_{r}\right), f\left(\mu_{b}\right), L$, and $S$. For a known structure, $L$ and $S$ are determined, and Poisson's ratio $\mu$ influences $[K]$ and displacement $\delta$ slightly. Therefore, $[K]$ is mainly influenced by $E_{c},\left(E_{r} / E_{c}\right)$, and $\left(E_{b} / E_{c}\right)$. We speculate that the displacements of the dam body and rock dam foundation are mainly influenced by $E_{c}$, $\left(E_{r} / E_{c}\right)$, and $\left(E_{b} / E_{c}\right)$.

Given the inversion model of $E_{c}, E_{r}$, and $E_{b}$ that is established on the basis of the spatial and temporal distributions of the displacement,

$$
\begin{aligned}
\delta_{H} & =f_{1}(H, x, y, z) \\
& =\mathrm{XF}[f(H), f(x, y, z)]+\mathrm{YG}[f(H), f(x, y, z)]+\mathrm{ZW}[f(H), f(x, y, z)] \\
& =X \sum_{i=0}^{m_{1}} \sum_{l, m, n=0}^{m_{3}} A_{1 \mathrm{ilmn}} H^{i} x^{l} y^{m} z^{n}+Y \sum_{i=0}^{m_{1}} \sum_{l, m, n=0}^{m_{3}} A_{2 \mathrm{ilmn}} H^{i} x^{l} y^{m} z^{n}+Z \sum_{i=0}^{m_{1}} \sum_{l, m, n=0}^{m_{3}} A_{3 \mathrm{ilmn}} H^{i} x^{l} y^{m} z^{n},
\end{aligned}
$$

where $\quad X=\left(E_{c 0} / E_{c}\right), \quad Y=\left(R_{0} / R\right), \quad R_{0}=\left(E_{r 0} / E_{c 0}\right)$, $R=\left(E_{r} / E_{c}\right), Z=\left(S_{0} / S\right), S_{0}=\left(E_{b 0} / E_{c 0}\right), S=\left(E_{b} / E_{c}\right)$, and $A_{1 \text { ilmn }}, A_{2 \text { ilmn }}$, and $A_{3 \text { ilmn }}$ are the coefficients in the fitting expressions of the spatial displacement fields caused by three hydraulic pressure-induced deformation components in the finite element simulation.

(1) Solving $E_{b}$

During the inversion analysis of $E_{b}$ based on the finite element simulation model for the position far away from the dam, the node displacement $\{\delta\}$ under certain hydraulic loads $\left\{R_{H}\right\}$ is mainly influenced by $E_{n}$ as follows:

$$
\{\delta\}=F\left(E_{b 1}, E_{b 2}, \ldots, E_{\mathrm{bM}}, H\right) .
$$

Then, the objective function is given by

$$
\begin{aligned}
Q= & \left(\left\{\delta_{i}\right\}-\left\{\bar{\delta}_{i}\right\}\right)^{T}\left(\left\{\delta_{i}\right\}-\left\{\bar{\delta}_{i}\right\}\right) \\
= & \left(\left\{\delta_{i}\right\}-F\left(E_{b 1}, E_{b 2}, \ldots, E_{\mathrm{bM}}, H\right)\right)^{T} \\
& \left(\left\{\delta_{i}\right\}-F\left(E_{b 1}, E_{b 2}, \ldots, E_{\mathrm{bM}}, H\right)\right) .
\end{aligned}
$$

Given that $f_{3}(\theta, x, y, z)$ and $f_{1}(H, x, y, z)$ are known, the following equation is true for a minimum $f_{2}(T, x, y, z)$ : 


$$
\frac{\partial Q}{\partial E_{\mathrm{bi}}}=0, \quad(i=1,2, \ldots, M)
$$

Therefore, the inversion value $\left\{E_{\mathrm{bi}}\right\}(i=1,2, \ldots, M)$ of $E_{b}$ could be obtained.

(2) Solving $E_{r}$

Supposing the displacement at $E_{r 0}$, which is calculated by the dam model under different water levels, is $\delta_{\mathrm{rH} 0}$; according to the inversion results of $E_{b}$, the displacement at this point, which is calculated by the large-scale finite element model under different water levels, is $\delta_{3 H}$. Next, the hydraulic pressure component $\left(\delta_{\mathrm{rH}}\right)$ at the measuring point is separated by the spatial displacement field model. $\delta_{\mathrm{rH}}$ minus $\delta_{3 H}$ is the real value of the rock foundation displacement $\delta_{\mathrm{rH}}$. Therefore, the objective function is established as follows:

$$
Q=\sum_{i=1}^{n}\left(\delta_{\mathrm{rH}_{i}}-\delta_{3 H_{i}}-\delta_{\mathrm{rH}_{0 i}}\right)^{2}=f\left(E_{r}\right)
$$

$Q$ is the objective function of inversion. Therefore, the following equation is true when $Q$ is the minimum value:

$$
\frac{\partial Q}{\partial E_{r}}=0
$$

\section{(3) Solving $E_{c}$}

The displacement data at the measuring points of the dam body include the displacements caused by the deformations of the dam body, dam foundation, and reservoir basin. Therefore, the displacements caused by the deformations of the rock foundation and reservoir basin are deducted from the hydraulic pressure $\left(\delta_{H}\right)$ separated from the spatial displacement field model. Supposing the concrete deformation modulus of the dam body is $E_{c 0}$ and the displacement at this point under different water levels is calculated by the finite element simulation model of the dam structure on the basis of the inversion result of $E_{r}$,

$$
Q=\sum_{i=1}^{n}\left[\left(\delta_{H}-\delta_{2 H}-\delta_{3 H}\right)-\left(\delta_{H}^{\prime}-\delta_{2 H}\right)\right]^{2}=f\left(E_{c}\right) .
$$

The following equation is true when $Q$ is the minimum:

$$
\frac{\partial Q}{\partial E_{c}}=0
$$

\section{QGA}

QGA innovatively introduces quantum calculation into the genetic algorithm and overcomes the difficulties of traditional genetic algorithms in the selection of the evolution mode. The approach satisfies the accuracy requirements of the fitness function through a more efficient and accurate intelligent population optimization.

The basic information unit in quantum calculation is the quantum state. The quantum state has three major characteristics in quantum calculation: quantum superposition, quantum entanglement, and quantum interference. Quantum is characterized by the state vector, and encoding genetic chromosome is characterized by the probability amplitude of quantum bit such that the superposition of multiple state vectors can be expressed by one piece of chromosome. Chromosome updating is realized by the quantum logic gate. The basic principle of QGA is introduced in the following text [20-27].

3.1. Quantum Bit Encoding. The QGA uses quantum bit as the physical medium to store unit information. Quantum bit is often formed by the superposition of two quanta. Quantum bit is obtained by

$$
|\varphi\rangle=\alpha|0\rangle \beta|1\rangle
$$

where $|0\rangle$ and $|1\rangle$ are the spinning downward state and spinning upward state, respectively; $\alpha$ and $\beta$ are the amplitude constants that satisfy $|\alpha|^{2}+|\beta|^{2}=1 ;|\alpha|^{2}$ is the probability of the spinning downward state; and $|\beta|^{2}$ is the probability of the spinning upward state.

Unlike traditional genetic algorithms, QGA expresses the gene state and stores gene state information by quantum bit encoding (0/1). For example, 1 means that the gene is at state " 1 ," and 0 means that the gene is at state " 0 ." If this gene contains uncertain information, this aspect could be expressed by the superposition of the " 0 " and " 1 " states. In other words, this gene state contains all possible information.

If an individual is composed of $m$ chromosomes, every individual could be expressed by

$$
q=\left[\begin{array}{cccc}
\alpha_{1} & \alpha_{2} & \cdots & \alpha_{m} \\
\beta_{1} & \beta_{2} & \cdots & \beta_{m}
\end{array}\right]
$$

3.2. Quantum Gate Updating. Quantum gate is the most basic operation of the quantum bit. Specific quantum gate operations are often selected according to specific problems. Multiple operations are generally optional. Quantum revolving gate is selected in this paper. The adjustment operation of the quantum revolving gate is

$$
U\left(\theta_{i}\right)=\left[\begin{array}{cc}
\cos \left(\theta_{i}\right) & -\sin \left(\theta_{i}\right) \\
-\sin \left(\theta_{i}\right) & \cos \left(\theta_{i}\right)
\end{array}\right]
$$

Quantum gate updating is

$$
\left[\alpha_{i}^{\prime} \beta_{i}^{\prime}\right]=U\left(\theta_{i}\right)\left[\begin{array}{l}
\alpha_{i} \\
\beta_{i}
\end{array}\right]=\left[\begin{array}{cc}
\cos \left(\theta_{i}\right) & -\sin \left(\theta_{i}\right) \\
-\sin \left(\theta_{i}\right) & \cos \left(\theta_{i}\right)
\end{array}\right]\left[\begin{array}{c}
\alpha_{i} \\
\beta_{i}
\end{array}\right],
$$

where $\left(\alpha_{i}, \beta_{i}\right)^{T}$ is the probability amplitude before and after updating the revolving gate of the $i^{\text {th }}$ quantum of the 




Figure 1: Coordinates of the revolving quantum gate.

chromosome and $\theta$ is the revolving angle. The revolving gate updating process is shown in Figure 1.

When the quantum bit is at the first and third quadrants, $\theta$ is positive ( $\theta$ rotates counterclockwise), which increases the probability of the " 0 " state, whereas a negative $\theta(\theta$ rotates clockwise) increases the probability of the " 1 " state. When the quantum bit is at the second and fourth quadrants, a positive $\theta$ raises the probability of the " 1 " state and a negative $\theta$ increases the probability of the " 0 " state.

3.3. Steps of QGA. The basic flowchart of QGA is shown in Figure 2.

(1) Population $Q\left(t_{0}\right)$ is initialized to produce the random chromosome encoded by quantum bits.

(2) Individuals in the initial population are measured singly, and the corresponding determined solutions of individuals are obtained as $P\left(t_{0}\right)$.

(3) The fitness degree of $P\left(t_{0}\right)$ is evaluated.

(4) The optimal individual and its fitness degree are recorded.

(5) The individual is judged whether it satisfies the end condition. If the end condition is satisfied, the step is exited; otherwise, the calculation is continued.

(6) If the individual does not satisfy the end condition, all the individuals in $Q\left(t_{0}\right)$ are measured again to calculate for the corresponding determined solutions.

(7) The fitness degrees of the different determined solutions are assessed.

(8) The individuals are adjusted by the quantum revolving gate $U(t)$, and a new population $Q(t+1)$ is attained.

(9) The optimal individual and its fitness degree are recorded.

(10) The iterations are increased by 1 , and the process returns to step (5).
3.4. Inversion Model Combining QGA and Finite Element Simulation. The commercial finite element software Abaqus involves a high-efficiency core solver and can establish the interface between Abaqus and MATLAB users through secondary development. Consequently, the inversion efficiency is increased. On the basis of the finite element simulation results, we construct an inversion model for QGA. The fitness function is established on the basis of the difference between the measured value and the finite element simulation result. The inversion of the mechanical parameters of hydraulic structural materials is realized by MATLAB programming. The specific steps are as follows:

(1) The calling interface of the Abaqus finite element program is established using MATLAB. The INP document of the Abaqus model and the constitutive Fortran subprogram document of materials are called through system commands. The problem is solved using the Abaqus finite element high-efficiency core.

(2) After the INP document is edited in step (1), the deformation data of the characteristic unit in the Abaqus finite element simulation document is generated using the Elprint command. The Fopen command in MATLAB is called, and the fitness function of the characteristic unit error is calculated.

(3) The QGA subprogram is compiled, and the fitness function of characteristic unit in step (2) is optimized.

(4) The inversion parameters are corrected in the command stream document using the Num2str command in the MATLAB language. Meanwhile, the revolving quantum gate is updated, and the next generation of population is obtained.

(5) Steps (1)-(4) are repeated until the objective function reaches the desired accuracy. The calculation flowchart of the inversion model combining the QGA and finite element simulation is shown in Figure 3. 


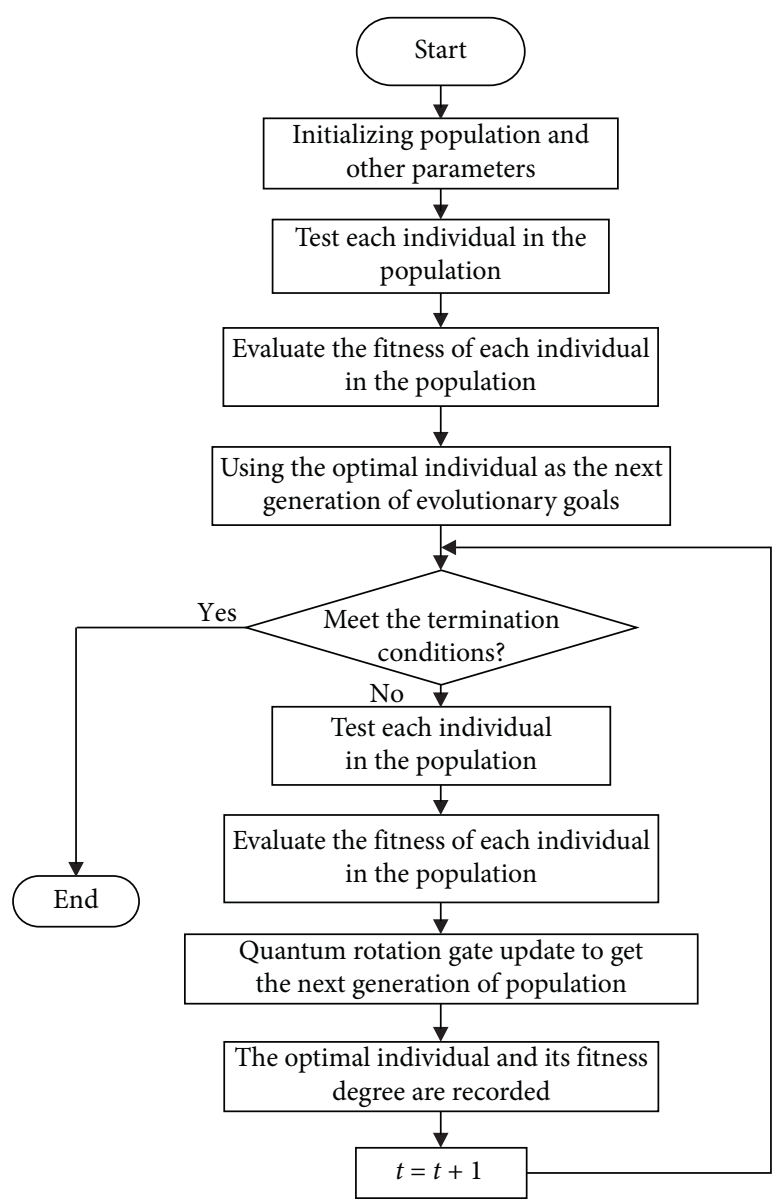

FIgURE 2: Flowchart of QGA.

\section{Case Study}

4.1. Introduction to the Engineering Project. The hydropower station located in the Yi Autonomous Prefecture of the Liangshan, Sichuan Province, was used as the case study in this work. It is the "control" reservoir gradient for the hydroelectric development planning at the midstream and downstream of the Yalung River. The station is mainly utilized for electricity generation and holds an outstanding downstream gradient compensation adjustment effect with its annual adjustment capability. The project belongs to the first-type first-class project, and the permanent major hydraulic structure is a first-class construction. The normal pool level, level of dead water, and the capacity below the normal pool level of the reservoir are $1880 \mathrm{~m}, 1800 \mathrm{~m}$, and 7.76 billion $\mathrm{m}^{3}$, respectively (Figure 4).

The dam is a concrete double-arch dam with a crest elevation of $1885.0 \mathrm{~m}$, and the elevation of the lowest foundation surface is $1580.0 \mathrm{~m}$. The maximum dam height, top width, foundation thickness, and thickness-height ratio are $305.0 \mathrm{~m}, 16.0 \mathrm{~m}, 63.0 \mathrm{~m}$, and 0.207 , respectively. The project achieved the main channel closure on December 4 , 2006, and has accomplished four stages of impounding since November 30, 2012.
The horizontal displacement of the dam body is monitored by normal/inverted vertical lines. A total of 57 measuring points were set at the left mountain; dam sections $\# 1, \# 5, \# 9, \# 11, \# 13, \# 16, \# 19$, and \#23; and crest elevations $(1885,1829,1778,1730,1664$, and $1601 \mathrm{~m})$ of the right mountain.

\subsection{Establishment and Portioning of the Finite Element Simulation Model of the High-Arch Dam}

4.2.1. Establishment of the Finite Element Simulation Model of the High-Arch Dam. The three-dimensional finite element simulation model of the high-arch dam in the hydropower station was constructed in accordance with its engineering design and geological data. The model covers 923,737 finite elements and 957,221 nodes. The dam body accounted for 29,840 finite elements and 36,079 nodes (Figure 5).

The calculation ranges of the model covered $900 \mathrm{~m}$ at the left and right banks from the central line of the arc dam; $750 \mathrm{~m}$ upstream and downstream from dam-crest origin; $700 \mathrm{~m}$ below the foundation surface; the scope from the rock slope above the crest elevation to the natural boundary elevation; the left and right mountains extended by 2.5 times 


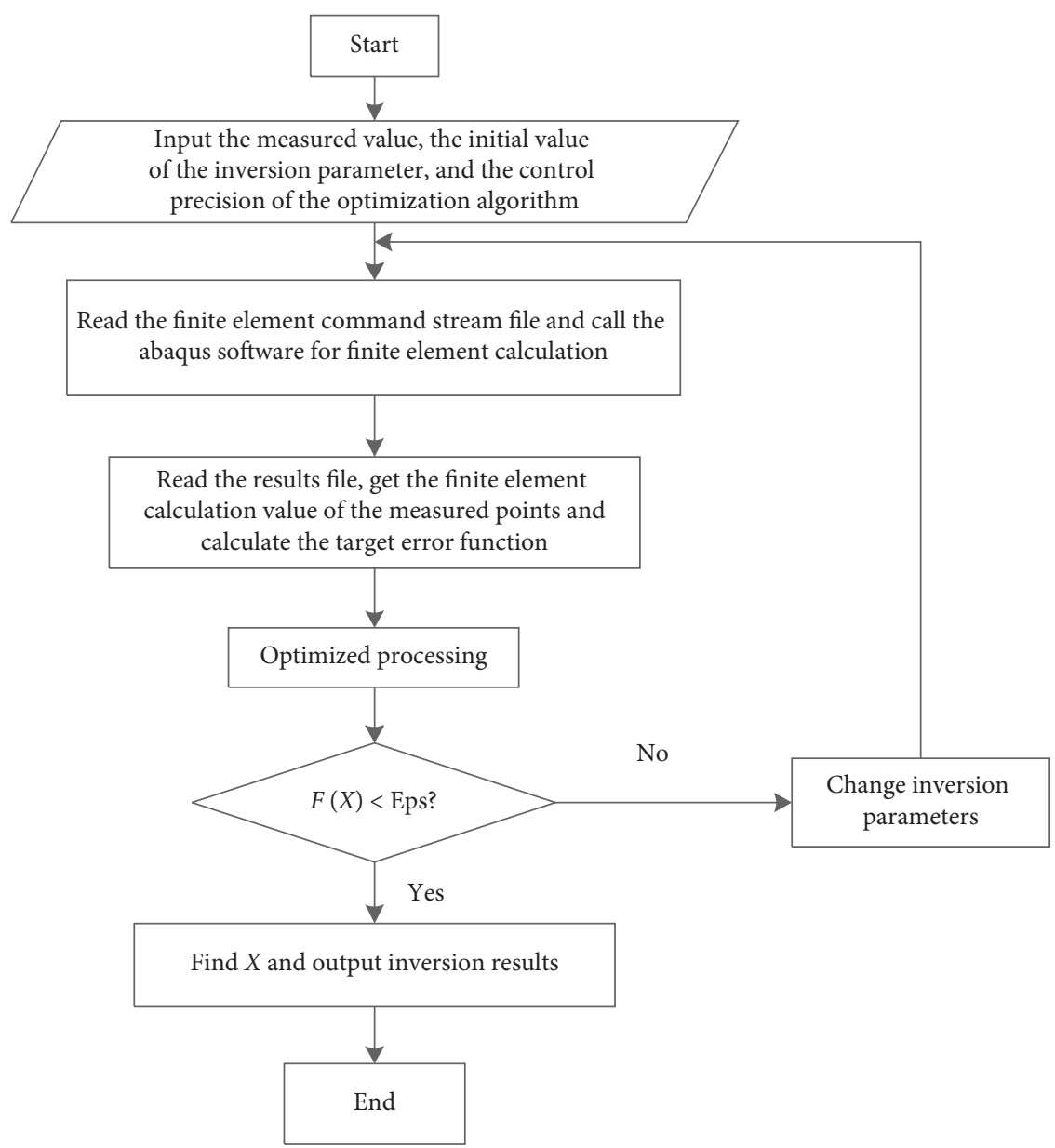

FIgURE 3: Flowchart of the inversion model combining QGA and finite element simulation.

the elevation toward upstream and downstream, respectively; and the crest elevation extended upward by one dam height. The rock mass conditions and mountain shapes at the two banks of the dam after excavation were simulated. The finite element model used hexahedral eight-node isoparametric elements. Considering the landform and geological conditions, some regions adopted pentahedron sixnode isoparametric elements.

\subsubsection{Partitioning of the Finite Element Model and Layout of} the Measuring Points. The three-dimensional finite element model renders the material portioning to the dam body and dam foundation consistent to those of actual conditions. The dam body was divided into $\mathrm{A}, \mathrm{B}$, and $\mathrm{C}$ regions. A total of 33 vertical-line measuring points were set in the \#5, \#9, \#11, \#13, \#16, and \#19 sections of the high-arch dam. The location of the measuring points and the corresponding material portioning is shown in Figure 6.
4.3. Inversion Results Analysis. An inversion analysis of the comprehensive deformation modulus of this high-arch dam was performed. The water levels on August 12, 2014 $(1858.34 \mathrm{~m})$, and August 24, $2014(1878.22 \mathrm{~m})$, were used as the calculation load of the finite element model and substituted into the finite element model for simulation. The inversion of the deformation modulus caused by the horizontal displacement at 32 measuring points in the three regions of the dam body was accomplished. The deformation inversion ranges were as follows: the deformation modulus range of region $A$ was $30-42 \mathrm{GPa}$, the deformation modulus range of region $\mathrm{B}$ was $30-42 \mathrm{GPa}$, and the deformation modulus range of region $\mathrm{C}$ was $25-37 \mathrm{GPa}$. The error fitness function was established on the basis of the differences between the measured horizontal displacement and the simulated horizontal displacement at different measuring points. The parameter inversion was implemented by QGA. Control parameters for the parameter inversion on the basis of the QGA were set as follows. The initial population was 




FIGURE 4: Layout chart of the high-arch dam.



FIGURE 5: Three-dimensional finite element model of the dam (upstream view).

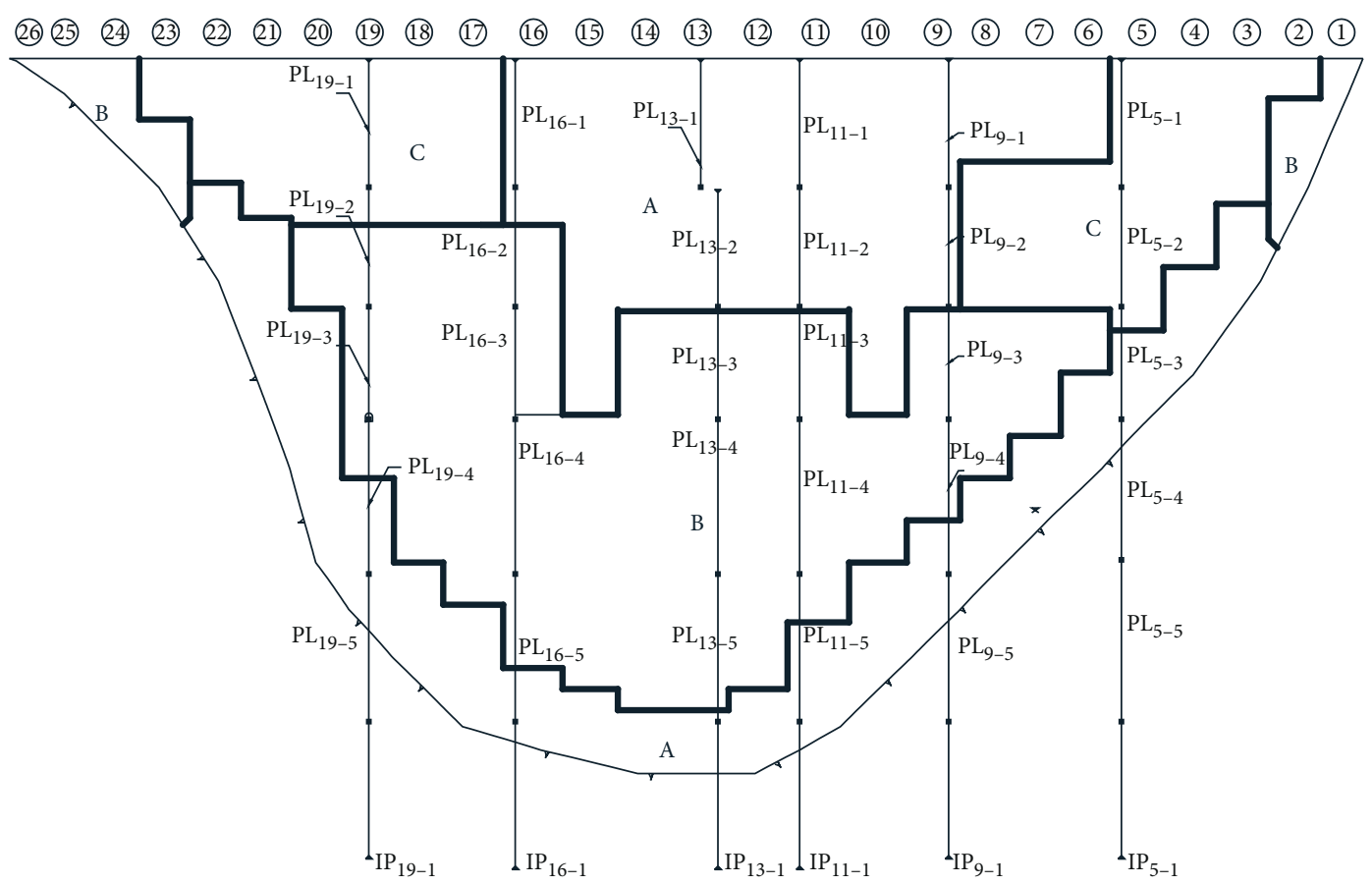

Figure 6: Distribution of the vertical-line measuring points and corresponding material partitioning. 
TABLE 1: Calculated results from inversion model by two optimization methods.

\begin{tabular}{llcccc}
\hline Method & Regions & Best searching value & Average searching value & Fastest searching time & Average searching time \\
\hline \multirow{3}{*}{ GA } & Region A & 39.37 & 37.11 & & 29326.9 \\
& Region B & 36.19 & 34.35 & & 34185.4 \\
& Region C & 32.09 & 30.84 & & \\
\multirow{2}{*}{ QGA } & Region A & 39.17 & 36.94 & 29105.7. & 25225.8 \\
& Region B & 34.98 & 34.18 & & \\
\hline
\end{tabular}

TABLE 2: Comparison of calculated and measured displacement values of the dam sections' measure points under the water level of $1858.34 \mathrm{~m}$

\begin{tabular}{|c|c|c|c|c|c|}
\hline \multirow{2}{*}{ Section } & \multirow{2}{*}{ Measuring point } & \multirow{2}{*}{ Altitude (m) } & \multirow{2}{*}{ Measured value (mm) } & \multicolumn{2}{|c|}{ Calculated value $(\mathrm{mm})$} \\
\hline & & & & GA & QGA \\
\hline \multirow{4}{*}{$5^{\#}$} & PL5-4 & 1729 & 2.72 & 2.70 & 2.71 \\
\hline & PL5-3 & 1778 & 4.86 & 4.79 & 4.82 \\
\hline & PL5-2 & 1829 & 5.29 & 5.21 & 5.23 \\
\hline & PL5-1 & 1885 & 18.46 & 18.04 & 18.14 \\
\hline \multirow{6}{*}{$9^{\#}$} & IP9-1 & 1601 & 2.13 & 2.13 & 2.13 \\
\hline & PL9-5 & 1665 & 7.60 & 7.46 & 7.49 \\
\hline & PL9-4 & 1729 & 17.86 & 17.17 & 17.33 \\
\hline & PL9-3 & 1778 & 19.45 & 18.28 & 18.92 \\
\hline & PL9-2 & 1829 & 14.64 & 14.09 & 14.22 \\
\hline & PL9-1 & 1885 & 6.22 & 5.95 & 6.01 \\
\hline \multirow{6}{*}{$11^{\#}$} & IP11-1 & 1601 & 8.35 & 7.93 & 8.03 \\
\hline & PL11-5 & 1664 & 23.14 & 22.48 & 22.63 \\
\hline & PL11-4 & 1730 & 33.49 & 30.12 & 31.96 \\
\hline & PL11-3 & 1778 & 30.88 & 28.83 & 29.95 \\
\hline & PL11-2 & 1829 & 22.42 & 21.33 & 21.58 \\
\hline & PL11-1 & 1885 & 14.67 & 13.84 & 14.03 \\
\hline \multirow{6}{*}{$13^{\#}$} & IP13-1 & 1601 & 6.83 & 6.67 & 6.71 \\
\hline & PL13-5 & 1664 & 27.68 & 25.94 & 26.34 \\
\hline & PL13-4 & 1730 & 32.42 & 29.69 & 31.18 \\
\hline & PL13-3 & 1778 & 27.08 & 25.17 & 26.21 \\
\hline & PL13-2 & 1829 & 18.96 & 17.80 & 18.07 \\
\hline & PL13-1 & 1885 & 31.08 & 29.64 & 29.97 \\
\hline \multirow{5}{*}{$16^{\#}$} & IP16-1 & 1601 & 11.93 & 11.54 & 11.63 \\
\hline & PL16-4 & 1664 & 29.61 & 26.88 & 28.37 \\
\hline & PL16-3 & 1730 & 24.61 & 22.15 & 23.49 \\
\hline & PL16-2 & 1778 & 16.26 & 15.45 & 15.89 \\
\hline & PL16-1 & 1829 & 9.28 & 8.94 & 9.02 \\
\hline \multirow{6}{*}{$19^{\#}$} & IP19-1 & 1601 & 3.64 & 3.62 & 3.63 \\
\hline & PL19-5 & 1664 & 11.88 & 11.41 & 11.52 \\
\hline & PL19-4 & 1730 & 24.56 & 23.85 & 24.01 \\
\hline & PL19-3 & 1778 & 22.24 & 21.45 & 21.88 \\
\hline & PL19-2 & 1829 & 17.60 & 16.70 & 17.19 \\
\hline & PL19-1 & 1885 & 9.70 & 9.53 & 9.58 \\
\hline
\end{tabular}

about $0.05-200$ times than that of the inversion parameters. The population size was 6000 , and the quantum bit was set to 25. The initial search superposed at equal probability, and the probability amplitude of the quantum bit was $\pm 2 \sqrt{2}$. The inversion results are shown in Table 1.
In order to check the computational efficiency and quality of quantum genetic algorithm (QGA), this paper also selects the traditional genetic algorithm (GA) to carry out inverse analysis on the deformation modulus of each partition of dam body. In order to compare the 
TABLE 3: Comparison of calculated and measured displacement values of the dam sections' measure points under the water level of $1878.22 \mathrm{~m}$.

\begin{tabular}{|c|c|c|c|c|c|}
\hline \multirow{2}{*}{ Section } & \multirow{2}{*}{ Measuring point } & \multirow{2}{*}{ Altitude (m) } & \multirow{2}{*}{ Measured value (mm) } & \multicolumn{2}{|c|}{ Calculated value $(\mathrm{mm})$} \\
\hline & & & & GA & QGA \\
\hline \multirow{4}{*}{$5^{\#}$} & PL5-4 & 1729 & 4.39 & 4.31 & 4.33 \\
\hline & PL5-3 & 1778 & 9.20 & 9.10 & 9.12 \\
\hline & PL5-2 & 1829 & 11.38 & 11.29 & 11.31 \\
\hline & PL5-1 & 1885 & 30.58 & 29.68 & 29.89 \\
\hline \multirow{6}{*}{$9^{\#}$} & IP9-1 & 1601 & 2.45 & 2.44 & 2.45 \\
\hline & PL9-5 & 1665 & 9.03 & 9.00 & 9.01 \\
\hline & PL9-4 & 1729 & 22.13 & 21.81 & 21.88 \\
\hline & PL9-3 & 1778 & 27.25 & 26.08 & 26.72 \\
\hline & PL9-2 & 1829 & 27.05 & 25.83 & 26.11 \\
\hline & PL9-1 & 1885 & 23.00 & 22.68 & 22.75 \\
\hline \multirow{6}{*}{$11^{\#}$} & IP11-1 & 1601 & 9.02 & 8.85 & 8.89 \\
\hline & PL11-5 & 1664 & 26.20 & 25.54 & 25.69 \\
\hline & PL11-4 & 1730 & 40.79 & 36.72 & 38.94 \\
\hline & PL11-3 & 1778 & 40.23 & 36.84 & 38.69 \\
\hline & PL11-2 & 1829 & 35.41 & 33.81 & 34.18 \\
\hline & PL11-1 & 1885 & 30.97 & 29.31 & 29.69 \\
\hline \multirow{6}{*}{$13^{\#}$} & IP13-1 & 1601 & 7.85 & 7.68 & 7.72 \\
\hline & PL13-5 & 1664 & 31.36 & 29.74 & 30.11 \\
\hline & PL13-4 & 1730 & 39.63 & 36.24 & 38.09 \\
\hline & PL13-3 & 1778 & 37.40 & 35.18 & 36.39 \\
\hline & PL13-2 & 1829 & 30.88 & 29.96 & 30.17 \\
\hline & PL13-1 & 1885 & 31.35 & 30.88 & 30.99 \\
\hline \multirow{5}{*}{$16^{\#}$} & IP16-1 & 1601 & 12.98 & 12.46 & 12.58 \\
\hline & PL16-4 & 1664 & 34.83 & 31.60 & 33.36 \\
\hline & PL16-3 & 1730 & 31.61 & 30.20 & 30.97 \\
\hline & PL16-2 & 1778 & 26.10 & 25.37 & 25.77 \\
\hline & PL16-1 & 1829 & 20.86 & 19.98 & 20.18 \\
\hline \multirow{6}{*}{$19^{\#}$} & IP19-1 & 1601 & 3.90 & 3.88 & 3.89 \\
\hline & PL19-5 & 1664 & 12.58 & 12.07 & 12.19 \\
\hline & PL19-4 & 1730 & 26.61 & 25.77 & 25.96 \\
\hline & PL19-3 & 1778 & 25.79 & 24.07 & 25.01 \\
\hline & PL19-2 & 1829 & 22.36 & 21.13 & 21.80 \\
\hline & PL19-1 & 1885 & 16.28 & 15.77 & 15.89 \\
\hline
\end{tabular}


FIgURE 7: The nephogram comparison between the calculated (QGA) displacement values and the measured values under $1858.34 \mathrm{~m}$.

computation abilities of these two algorithms equally, the two methods are calculated for 50 times, respectively. The calculation results are shown in Table 1, from which the following is obtained:

(1) Since the grouping quantum genetic algorithm uses quantum probability coding to enhance the population diversity, the search average of the grouping quantum genetic algorithm is slightly different from the GA average. However, the grouping quantum genetic algorithm can effectively avoid the GA method's local minimum weakness.

(2) In terms of the computational efficiency of the optimization algorithm, there is little difference between the two methods in the fastest search time, but the average searching time of using QGA is shorter, which greatly improves the operation efficiency. 

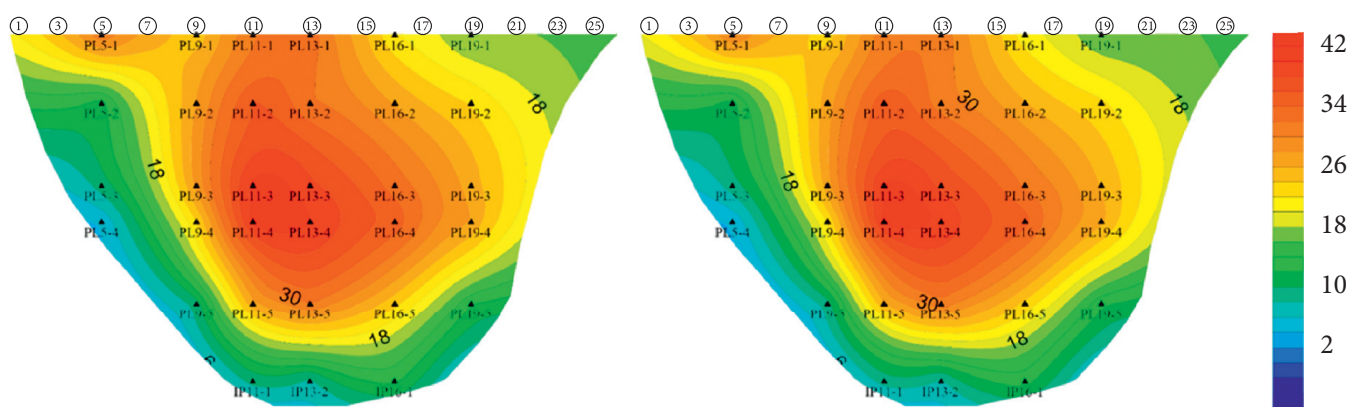

FIGURE 8: The nephogram comparison between the calculated (QGA) displacement values and the measured values under $1878.22 \mathrm{~m}$.

To verify the accuracy of the inversion results, the inversion results of the deformation modulus at different regions were used as the calculation parameters. Given the finite element analysis, the calculated and measured radial displacements at the vertical-line measuring points under different hydraulic loads (the water level of $1858.34 \mathrm{~m}$ and $1878.22 \mathrm{~m}$ ) were obtained through finite element analysis (Tables 2 and 3).

The comparison of nephograms between the calculated (by QGA) displacement values and the measured values are as follows (Figures 7 and 8).

Tables 2 and 3 show that the partitioning inversion of the deformation modulus achieves similar simulated and measured radical displacements at different vertical-line measuring points for the same dam body under different hydraulic loads. Moreover, a relative error of less than $5 \%$ is revealed.

From Figures 7 and 8, the differences between the calculated displacement of inverted parameters and the measured displacement are small, which means the inversion results are reasonable and credible.

The calculated results of the dam body deformation modulus from the FEM based on QGA are closer to the measured dam body horizontal displacement and have higher computational accuracy than the inversion results of the traditional genetic algorithm (GA). The results show that the quantum genetic algorithm (QGA), with better accuracy and computational efficiency, can find the better individuals faster, and the calculation process does not depend on the initial population.

\section{Conclusion}

On the basis of the conventional inversion algorithms of the physical and mechanical parameters of dam deformation, this paper focuses on studying the partitioning inversion analysis algorithm of the deformation modulus of a higharch dam and establishes the objective function for the corresponding partitioning inversion of the deformation modulus of the dam body. The inversion analysis steps are introduced. Several conclusions are drawn:

(1) Given the most fundamental equilibrium equation, the characterization method of the dam displacement field under hydraulic loads is studied. On this basis, the inversion algorithm of the deformation modulus of the dam body, dam foundation, and reservoir basin is discussed using the spatial-temporal safety monitoring model of the spatial displacement field. The inversion formulas of the mean deformation modulus of the dam body, dam foundation, and reservoir basin are deduced.

(2) Multioptimization of the solution space is accomplished through QGA, which increased the chromosome diversity and ensured the algorithm convergence at the global optimal solution. Compared with traditional genetic algorithm, this aspect holds the characteristics of quick convergence and high conversion accuracy.

(3) The fitness function is established on the basis of the difference between the measured and simulated results by combining the QGA and finite element simulation. The comprehensive deformation modulus of the different regions of the high-arch dam is deduced in reverse. The inversion results are used in the threedimensional finite element calculation of the dam body and are compared with the measured data. The result demonstrates that the inversion results can represent the comprehensive deformation modulus of different dam body regions. This finding verifies the validity and practical application value of the proposed partitioning inversion algorithm.

\section{Data Availability}

The monitoring data of the high-arc dam used to support the findings of this study are available from the corresponding author upon request.

\section{Conflicts of Interest}

The authors declare that there are no conflicts of interest regarding the publication of this paper.

\section{Authors' Contributions}

Yantao Zhu wrote the original draft and reviewed and edited the manuscript. Xinqiang Niu supervised the study. Chongshi $\mathrm{Gu}$ administrated the project and formulated methodology. Jimin Wang obtained resources. Erfeng Zhao performed data curation. Lixian Huang did formal analysis. All authors have read and agreed the published version of the manuscript. 


\section{Acknowledgments}

This work was supported by the National Key R\&D Program of China (Grant no. 2018YFC0407104), the National Natural Science Foundation of China (Grant nos. 51739003, 51909173, 52079046, 51979093, and 51779086), the Fund of National Dam Safety Research Center (CX2018Z38), Free exploration project of Hohai University (no. B200201058), Project Funded by the Priority Academic Program Development of Jiangsu Higher Education Institutions (YS11001), Basic Research Project Funded by the National Key Laboratory (nos. 20195025912 and 520003812), Open Foundation of Changjiang Survey, Planning, Design and Research Co., Ltd. (no. CX2019K01).

\section{References}

[1] Y. Xiang, D. Zheng, X. He et al., "Physical and mechanical parameters inversion based on MSC.Marc," Energy and Hydropower Science, vol. 21, no. 4, pp. 7-10, 2003.

[2] Li Bo, B. Xu, J. Wu et al., "Inversion of dam mechanical parameters based on least squares support vector machine," Chinese Journal of Geotechnical Engineering, no. 11, pp. 1722-1725, 2008.

[3] B. Xu, R. Pang, and Y. Zhou, "Verification of stochastic seismic analysis method and seismic performance evaluation based on multi-indices for high CFRDs," Engineering Geology, vol. 264, Article ID 105412, 2020.

[4] j. Chen, "Analysis report on prototype observation data of liujiaxia gravity dam," East China institute of water resources, vol. 4, 1981.

[5] $\mathrm{Z} . \mathrm{Wu}$ and h. Ruan, "Inverse analysis of observation data of concrete dam," Journal of Hohai University, vol. 2, pp. 10-17, 1989.

[6] C. Gu and $\mathrm{Z}$. Wu, "Inversion of viscosity coefficient $\mathrm{n}$ of dam and rock base," Journal of Hydraulic Engineering, vol. 30, no. 8, pp. 11-15, 1999.

[7] H. Su and L. Peng, "Inverse analysis method of concrete dam material parameter interval," Journal of Hohai University, vol. 36, no. 5, pp. 654-658, 2008.

[8] M. I. Friswell, J. E. T. Penny, and S. D Garvey, "A combined genetic and eigensensitivity algorithm for the location of damage in structures," Computers and Structures, vol. 69, no. 5, pp. 547-556, 1998.

[9] S. Li, Y. Liu, and D. Wang, "Nonlinear inversion of aquifer parameters based on simulated annealing," Journal of Xi'an Jiao Tong University, vol. 35, no. 5, pp. 546-548, 2001.

[10] S. Li, Y. Liu, and D. Wang, "The inversion of initial stress field of rock mass based on genetic algorithm," Journal of China Coal Society, vol. 26, no. 1, pp. 13-17, 2001.

[11] X. Guo, C. Wang, and $\mathrm{H}$. Wu, "Nonlinear finite element analysis of rockfill dam with asphalt concrete core wall," Journal of Applied Science and Engineering Science, vol. 58, pp. 19-24, 1995.

[12] W. Xiang, H. Su, and Z. Wu, "Inversion of physical and mechanical parameters based on dam safety monitoring data," Journal of Hydraulic Engineering, pp. 98-102, 2004.

[13] Y. Xiang, H. Su, and C. Gu, "Inversion of physical and mechanical parameters based on genetic algorithm," Proceedings of the Yangtze River Academy of Sciences, vol. 20, pp. 55-58, 2003.

[14] J. Niu and K. Shi, "Study on interlayer stress of RCC gravity dam,” Hydraulic Power, vol. 32, pp. 70-71, 2006.
[15] 1. Zhao and y. Dong, "Hybrid particle swarm optimization algorithm based on quantum genetics," Computer Engineering and Design, vol. 35, no. 7, pp. 2566-2577, 2014.

[16] c. Liang, B. hua, m. Cai et al., "Research progress of quantum genetic algorithm," Computer Applied Research, no. 7, pp. 2401-2405, 2012.

[17] z. Wang, b. Yang, x. Lv et al., "Research on an improved quantum genetic algorithm," Journal of $X i$ 'an University of Technology, vol. 28, no. 2, pp. 145-151, 2012.

[18] b. Xu, z. Peng, and j. Yu, "An improved quantum genetic algorithm based on cloud model," Computer Applied Research, vol. 28, no. 10, pp. 3684-3686, 2011.

[19] B. Chen, X. Fu, X. Guo et al., "Zoning elastic modulus inversion for high arch dams based on PSOGSA-SVM method," Advances in Civil Engineering, vol. 2019, Article ID 7936513, 13 pages, 2019.

[20] K. H. Han and J. H. Kim, "Quantum-inspired evolutionary algorithm for a class of combinatorial," IEEE Transactions on Evolutionary Computation, vol. 6, pp. 580-593, 2002.

[21] K.-H. Han and J.-H. Kim, "Quantum-Inspired evolutionary algorithms with a new termination criterion," IEEE Transactions on Evolutionary Computation, vol. 8, pp. 156-169, 2004.

[22] C. Liang, H. Bai, M. Cai et al., "Research progress of quantum genetic algorithm," Research and Application of Computer, vol. 29, no. 7, pp. 2401-2405, 2012.

[23] W. Sun and Y. He, "Analysis of the improved genetic algorithm of concrete thermal parameters of cushion layer inversion and the causes of early cracks," Journal of Wuhan University: Engineering Science, no. 2, pp. 193-200, 2014.

[24] Z. Lu and M. Gao, "Parameter optimization of support vector machine based on improved genetic algorithm," The Network of New Media Technology, vol. 31, no. 5, pp. 11-15, 2010.

[25] J. Yang, Li Yang, J. Li et al., "Improved genetic algorithm partial least squares regression model of dam deformation monitoring," Journal of Northwest Agriculture and Forestry University: Natural Science Edition, vol. 38, no. 2, pp. 206-210, 2010.

[26] Q. Dong, W. Zhou, Li Yun et al., "The improved genetic algorithm in Jinghuiqu Irrigation Area hydrogeological parameters in application," Chinese Rural Water Conservancy and Hydroelectric Power, no. 5, pp. 27-30, 2014.

[27] H. GuoW. Jin et al., et al., "Group quantum genetic algorithm and its application," Journal of Southwest University of Science and Technology: Natural Science Edition, vol. 19, no. 1, pp. 18-21, 2004. 